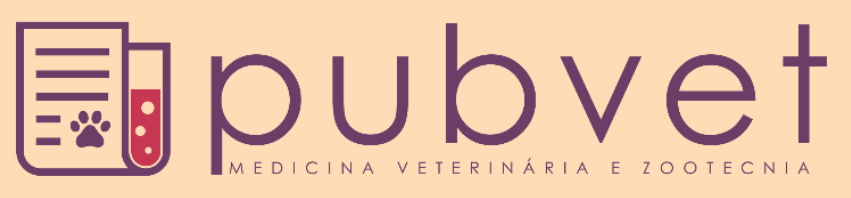

https://doi.org/10.31533/pubvet.v15n07a863.1-10

\title{
Degeneração mixomatosa de valva mitral em cães: Revisão
}

\author{
Caroline de Oliveira Silva Almeida ${ }^{1 * \bullet}$ \\ ${ }^{1}$ Graduanda de Medicina Veterinária da Pontifícia Universidade Católica de Campinas. Campinas, São Paulo, Brasil. \\ *Autor para correspondência, E-mail: caroline.oliveira2014@gmail.com
}

Resumo. A degeneração mixomatosa de valva mitral é uma afecção que acomete diversos animais, majoritariamente os cães de pequeno porte, raça definida, machos e idosos. Como se trata de uma das principais cardiopatias que afeta esses canídeos, objetivou-se compilar as informações mais relevantes e recentes na literatura sobre este tema, o que foi feito utilizando bases de dados e livros. Embora a etiologia não tenha sido totalmente esclarecida até o momento da conclusão deste trabalho, há evidências científicas que corroboram a correlação entre a ativação de mecanismos de proliferação celular cardíaco da fase embrionária com o desenvolvimento da degeneração. O diagnóstico pode ser realizado por meio da anamnese, exame físico, de imagem, bioquímicos e eletrocardiograma. A partir destes e dos sinais clínicos, pode-se estadiar a doença e avaliar as opções terapêuticas para então conduzir o tratamento da forma adequada. Por se tratar de uma desordem de caráter crônico que resulta em modificações na conformação estrutural de componentes cardíacos, o tratamento clínico não é curativo. Sendo assim, diversos fármacos podem ser administrados com o objetivo de inibir os mecanismos compensatórios deletérios ao organismo do indivíduo como forma de promover maior expectativa e qualidade de vida. O tratamento cirúrgico pode ser realizado utilizando diferentes técnicas, mas são encontrados diversos impedimentos para a realização na rotina em Medicina Veterinária.

Palavras chave: Cães, cardiopatia, degeneração, mitral

\section{Myxomatous mitral valve disease in dogs: Review}

\begin{abstract}
Myxomatous mitral valve degeneration is a affection that affects several animals, mainly of small breed animals, male and elderly. As it is one of the main cardiopathy that affect these canids, the objective was to compile the most relevant and recent information in the literature on this topic, which was done using databases and books. Although the etiology was not determined until the conclusion of this work, there is scientific evidence that corroborates the correlation between the activation of cardiac cell proliferation mechanisms in the embryonic phase with the development of degeneration. The diagnosis of which can be made through anamnesis, physical examination, imaging, biochemicals and electrocardiogram. Based on these and clinical signs, we can stage the disease and evaluate therapeutic options to conduct the treatment properly. Because it is a chronic disorder that results from changes in the structural conformation of cardiac components, clinical treatment is not curative. Therefore, several drugs can be administered in order to inhibit the harmful compensatory mechanisms to the individual's organism as a way to promote greater life expectancy and quality. Surgical treatment can be performed using different techniques, but found several impediments to the performance of this type of procedure in the routine in Veterinary Medicine.
\end{abstract}

Keywords: Cardiopathy, degeneration, dogs, mitral 


\section{Degeneración mixomatosa de la válvula mitral en perros: Revisión}

Resumen. La degeneración mixomatosa de la válvula mitral es una condición que afecta a varios animales, principalmente perros pequeños, razas definidas, machos y ancianos. Al tratarse de una de las principales cardiopatías que afecta a estos cánidos, el objetivo fue recopilar la información más relevante y reciente de la literatura sobre este tema, lo cual se realizó mediante bases de datos y libros. Aunque la etiología no ha sido completamente aclarada hasta la conclusión de este trabajo, existe evidencia científica que corrobora la correlación entre la activación de los mecanismos de proliferación celular cardíaca en la fase embrionaria con el desarrollo de la degeneración. El diagnóstico se puede realizar mediante anamnesis, exploración física, imagenología, bioquímica y electrocardiograma. En función de estos y los signos clínicos, es posible estadificar la enfermedad y evaluar las opciones terapéuticas para realizar el tratamiento en consecuencia. Debido a que es un trastorno crónico que produce cambios en la conformación estructural de los componentes cardíacos, el tratamiento clínico no es curativo. Así, se pueden administrar varios fármacos con el fin de inhibir los mecanismos compensatorios dañinos al organismo del individuo como una forma de promover una mayor expectativa de vida y calidad de vida. El tratamiento quirúrgico se puede realizar mediante diferentes técnicas, pero existen varios impedimentos para el desempeño rutinario en Medicina Veterinaria.

Palabras clave: Perros, cardiopatía, degeneración, mitral

\section{Introdução}

A Degeneração Mixomatosa de Valva Mitral (DMVM) é uma das cardiopatias mais importantes na medicina veterinária de pequenos animais, visto que acomete um grande número de cães, com maior ocorrência entre os de raça pequena, machos e idosos. Nos últimos anos, associou-se o desenvolvimento da cardiopatia em questão com eventos semelhantes aos da embriologia da valva que ocorrem em indivíduos adultos e se atualizou os conceitos de diagnóstico, estadiamento e tratamento da afecção em cães com o consenso publicado pelo Colégio Americano de Medicina Interna Veterinária.

Dessa forma, esta revisão buscou compilar as informações mais recentes publicadas em jornais e revistas acadêmicos, bem como as dispostas nos livros.

\section{Degeneração mixomatosa de valva mitral em cães}

As doenças cardíacas representam 10\% dos atendimentos primários, sendo $75 \%$ delas compatíveis com a degeneração mixomatosa de valva mitral, também denominada endocardiose valvular (Keene et al., 2019).

Um estudo realizado na Inglaterra por Lopez-Alvarez et al. (2015), baseado em dados obtidos de 97 clínicas a respeito de 111.967 cães com mais de um ano de idade, dentre os quais 405 apresentaram diagnóstico de DMVM, concluiu que houve maior prevalência da doença em machos, bem como nas seguintes raças: Cavalier King Charles Spaniel (CKCS), King Charles Spaniel, Whippet, Poodle, Yorkshire Terrier, Shih Tzu, Chihuahua, Miniature Schnauzer, Dachshund, Border Collie, Lhasa Apso e Cocker Spaniel, West Highland White Terrier e Jack Russell Terrier. Ainda sobre o estudo conduzido na Inglaterra, determinou-se que 78,02\% das raças possuíam menos de $20 \mathrm{~kg}$, e a idade média dos indivíduos acometidos foi entre oito e 13 anos, sendo a ocorrência da degeneração diretamente proporcional a idade.

Apesar dos indivíduos mais pesados não serem tão acometidos como os de raças pequenas, de acordo com Borgarelli et al. (2004), em uma análise de 58 pastores alemães e 49 cães com menos de $15 \mathrm{~kg}$, os animais com maior peso apresentaram maior predisposição a determinadas arritmias, o que acarreta em pior prognóstico.

\section{Etiologia}

O modo como ocorre a DMVM ainda não está totalmente esclarecido, tanto na medicina humana quanto na veterinária. Entretanto, nos últimos anos, correlacionou-se positivamente alterações na 
transformação sofridas durante a embriogênese cardíaca com a causa da doença no indivíduo adulto como consequência de fatores genéticos, mecânicos ou sistêmicos (Aikawa et al., 2019; Oyama et al., 2020). Uma das explicações para o acometimento de raças com tamanho menor em detrimento das grandes e gigantes poderia ser a relação dos genes que determinam o porte do animal, compartilhado entre elas. Junto desses genes, podem estar presentes os chamados genes ligados que podem estar correlacionados com o desenvolvimento da afecção citada, visto que estão envolvidos no desenvolvimento cardíaco (Parker \& Kilroy-Glynn, 2012).

Buscou-se correlacionar a genética com o desenvolvimento de DMVM utilizando cães da raça CKCS em uma análise conduzida por Madsen et al. (2011), na qual se avaliou a presença de dois locus genéticos, os quais podem estar correlacionados com o desenvolvimento da degeneração.

Os estudos mais recentes apontam a hipótese de que as células endoteliais valvares, a partir da ação da serotonina e TNF-Beta, regulado por genes, citados anteriormente, sofram transição mesenquimal, ativando as células intersticiais, que por sua vez aumentam as quantidades de enzimas proteolíticas e mediadores inflamatórios (Oyama et al., 2020; Rabkin et al., 2001; Tan et al., 2019). A partir dessas transformações, ocorre alteração da matriz extracelular do tecido valvar a partir da deposição de proteoglicanos e glicosaminoglicanos na camada esponjosa e desorganização das fibras colágenas na camada fibrosa dos folhetos valveres e nas cordoalhas tendíneas que caracteriza o tecido conjuntivo mixomatoso (Fox, 2012; Rabkin et al., 2001; Richards et al., 2012). Entretanto, até o período deste trabalho, não foram estabelecidos quais mecanismos estão envolvidos na apresentação da doença em questão, apenas que possivelmente se trata de uma alteração hereditária e distúrbios na produção e metabolização de serotonina ou modificações nos receptores desta substância de forma conjunta à alteração da expressão de TNF - Beta, que desencadeia alterações estruturais na valva mitral (Markby et al., 2020; Meurs et al., 2018; Oyama \& Levy, 2010; Oyama et al., 2020).

\section{Estadiamento}

No ano de 2019 foi publicado um novo guideline para o estadiamento da DMVM pelo Colégio Americano de Medicina Interna Veterinária (ACVIM), que possui maior acurácia ao associar os achados clínicos com as alterações morfológicas, visando adotar a conduta ideal para cada estágio (Keene et al., 2019). Os estágios da doença são divididos em A, B1, B2, C e D. Para que o animal se enquadre no primeiro, deverá apresentar predisposição genética elevada à doença, como é o caso das raças Cavalier King Charles Spaniel (Keene et al., 2019).

Para se enquadrar no estágio B, o animal deve ser assintomático, apresentar sopro cardíaco em foco de mitral e sinais estruturais de degeneração valvar ao exame ecocardiográfico. Quando houver ausência de repercussão hemodinâmica, ou seja, medida do ventrículo esquerdo normalizado pelo peso dentro dos parâmetros normais, expressas na tabela 1, o animal deverá ser classificado como B1 (Keene et al., 2019).

Tabela 1. Um dos 4 critérios que identificam o estágio B2 em cães é o volume ventricular normalizado pelo peso, identificados abaixo.

\begin{tabular}{ll|ll}
\hline PC, $\mathrm{kg}$ & LVIDD, $\mathrm{cm}$ & PC, $\mathrm{kg}$ & LVIDD, cm \\
\hline 1 & 1,7 & 11 & 3,4 \\
2 & 2,1 & 12 & 3,5 \\
3 & 2,4 & 13 & 3,6 \\
4 & 2,6 & 14 & 3,7 \\
5 & 2,7 & 15 & 3,8 \\
6 & 2,9 & 16 & 3,8 \\
7 & 3,0 & 17 & 3,9 \\
8 & 3,1 & 18 & 4,0 \\
9 & 3,2 & 19 & 4,0 \\
10 & 3,3 & 20 & 4,1 \\
\hline
\end{tabular}

Fonte: adaptado de Keene et al. (2019).

Quando houver repercussão hemodinâmica, as medidas para o ventrículo esquerdo estarão acima do ideal, portanto o animal será classificado como B2. Outros fatores determinantes para essa classificação são: relação entre aorta e átrio esquerdo maior que 1,6; sopro em foco de mitral de intensidade 3 de 6; vertebral heart size (VHS) acima do normal de acordo com a raça, de modo geral, acima de 10,5 (eene et al., 2019). 
Para classificação em estágio $\mathrm{C}$, o animal precisa apresentar todas as alterações vistas em B2 associadas a um quadro de insuficiência cardíaca, ou seja, incapacidade do coração em bombear volume de sangue adequado capaz de atender todas as demandas metabólicas em condições fisiológicas. Nesta classificação, o indivíduo apresenta sintomatologia e ainda não é refratário às medicações usuais indicados para a comorbidade em questão (Keene et al., 2019).

A presença das alterações vistas em $\mathrm{C}$ correlacionadas com a refratariedade aos tratamentos convencionais, o animal é classificado como D. Dessa forma, são necessárias doses acima das utilizadas em outros estágios para evitar colapso do sistema cardiovascular e pulmonar (Keene et al., 2019).

\section{Diagnóstico}

Os sinais apresentados pelos animais acometidos pela endocardiose valvular são inespecíficos. Em muitos casos, os pacientes podem passar anos sem qualquer alteração clínica perceptível ao tutor, geralmente estes são os indivíduos cujo estadiamento é de grau leve a moderado (Ettinger et al., 2002).

Os sinais clínicos relatados pelos tutores da anamnese podem ser: intolerância ao exercício físico; síncope; tosse seca, decorrente da compressão dos brônquios principais pelo átrio esquerdo ou pelo edema pulmonar; caquexia; síncope; hiporexia (Cunningham, 2011; Ettinger et al., 2002; Häggström et al., 2016; Kim et al., 2017; King \& Boag, 2007; Lopez-Alvarez et al., 2015). Ao exame físico, por meio da auscultação, é possível encontrar arritmias, sons de crepitação em topografia de pulmão, expulsão de secreção espumosa pela via aérea superior, indicando edema pulmonar; indícios de insuficiência cardíaca direita secundária, como a ascite, hepatomegalia, efusão pleural, edema de membros e prepúcio bem como pulso jugular positivo; mucosas cianóticas ou pálidas, dispneia, taquipneia durante o repouso ou prolongada após a realização de exercícios físicos, sendo estes sinais comumente associados a hipertensão pulmonar secundária à afecção; abafamento de sons cardíacos na presença de derrame pericárdico ou pleural, este também caracterizado por alteração na percussão de tórax e sopro cardíaco em foco de valva mitral durante a fase sistólica, que poderá ser graduado de um a seis (Boswood et al., 2016; Ettinger et al., 2002; Häggström et al., 2016; King \& Boag, 2007; Reinero et al., 2020).

\section{Exames de imagem}

A radiografia de tórax é indicada como triagem nos cães com suspeita clínica de DMVM, com a finalidade de observar se há alteração em parênquima pulmonar bem como aumento de silhueta cardíaca (Kealy et al., 2012; King \& Boag, 2007). Deverão ser realizadas ao menos duas projeções, sendo uma laterorateral (LL) e outra ventro-dorsal (VD) ou dorso-ventral (DV), sendo esta última preterida em detrimento da primeira, dada a possibilidade de impressões errôneas de cardiomegalia (Kealy et al., 2012).

A avaliação do tamanho do contorno cardíaco poderá ser feita utilizando o método VHS, elucidado na Figura 1, obtido em projeção LL (Buchanan, 2000).

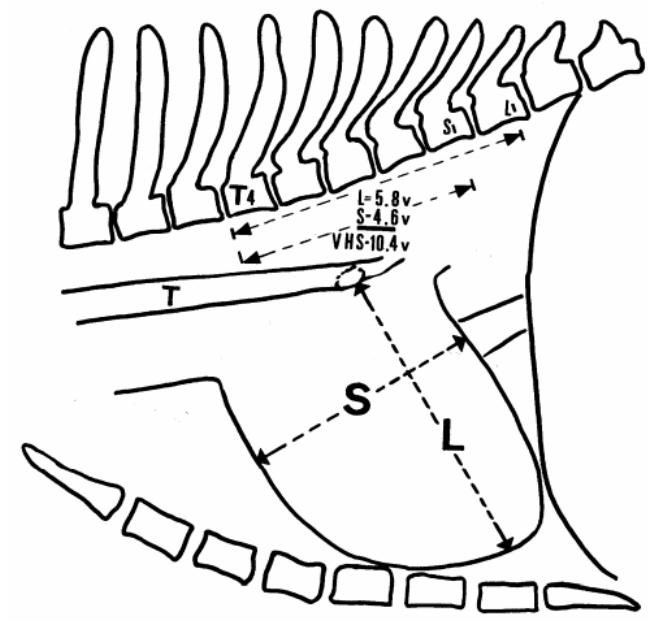

Figura 1. Método VHS de mensuração cardíaca. Fonte: Buchanan (2000). 
Outra medida com maior acurácia para detecção da DMVM e progressão da mesma é a Dimensão Radiográfica do Átrio Esquerdo (RLAD), medida obtida na mesma projeção que a descrita anteriormente a partir de uma linha traçada da carina da traqueia ao átrio esquerdo na interseção dos eixos L e S do VHS, de modo a avaliar quantos espaços intervertebrais a mesma ocupa (Malcolm et al., 2018). Estudos demonstraram forte correlação entre o a medida RLAD e a relação átrio esquerdo $\backslash$ aorta, obtida pela ecocardiografia, e dessa forma ela poderá ser utilizada de forma complementar para avaliar a presença de doenças que levem a dilatações atriais, como a afecção em questão (Salguero et al., 2019).

Outro método radiográfico para avaliação de tamanho de átrio esquerdo, descrito no consenso de diagnóstico e tratamento da DMVM, é tamanho vertebral do átrio esquerdo (VLAS), mensurado a partir de uma reta traçada da carina da traqueia à porção dorsal da veia cava caudal (Keene et al., 2019; Malcolm et al., 2018). Entretanto, mesmo com as novas técnicas de avaliação, o método diagnóstico referido avalia tamanho e formato cardíacos, sendo o ecocardiograma, considerado padrão ouro para o diagnóstico da DMVM, essencial para análise funcional, o que inclui o funcionamento da valva mitral, e de todas as estruturas do coração (Chetboul \& Tissier, 2012; Kealy et al., 2012). O posicionamento ideal para a realização do exame é o decúbito lateral esquerdo e direito, podendo ser feito com o animal em estação caso este seja muito grande ou se apresente dispneico (Boon, 2011).

Os achados variam de acordo com a severidade da afecção, sendo comum a todos os estágios a regurgitação mitral (RM), visualizada no modo Doppler colorido em graus variados, espessamento e prolapso dos folhetos valvares, com destaque para a região anterior, e presença de nódulos nas bordas destes (Boswood et al., 2016; Terzo et al., 2009).

\section{Bioquímicos}

Podem ser utilizados em associação aos exames de imagem, visto que trazem informações concernentes à presença de comprometimento cardíaco e ao prognóstico (Oyama, 2013). Dentre os marcadores de lesão cardíaca, os que merecem destaque pela confiabilidade evidenciada por estudos são os peptídeos natriuréticos (PN) e as troponinas, associados, respectivamente, à sobrecarga dos miócitos e morte dos mesmos (Boswood et al., 2016; Langhorn \& Willesen, 2016; Lima \& Ferreira, 2017; Oyama, 2013).

Os PN de importância diagnóstica são os ANP (peptídeo natriurético atrial) e o BNP (peptídeo natriurético cerebral), produzidos nos miócitos e liberado em condições de estiramento excessivo das câmaras cardíacas para promoção da natriurese, diurese e inibição dosistema renina-angiotensinaaldosterona, como forma de reduzir a volemia (Braunwald, 2005; Cunningham, 2011).

Tais biomarcadores podem ser identificados no sangue do animal por um curto período, e os níveis séricos destes, embora não sejam consideravelmente úteis no estadiamento, podem predizer se o animal apresenta cardiopatia em estágios assintomáticos ou ICC, de modo a excluir o diagnóstico de doença pulmonar primária (Eriksson et al., 2014; Oyama, 2013; Wolf et al., 2013).

Com relação às troponinas, estas se encontram nos miócitos e exercem a função na contração do músculo cardíaco, portanto, quando há morte dessas células, ocorre liberação desse componente, que pode ser identificado em uma amostra de sangue (Boswood et al., 2016).

No caso da afecção em questão, estudos conduzidos por (Borgarelli et al., 2004) utilizando 81 cães de raças pequenas, diferentes sexos e estágios da DMVM, demonstraram que houve incremento das concentrações da proteína citada no parágrafo anterior, com maior relevância nos animais com doença moderada a severa.

\section{Eletrocardiograma e pressão arterial}

O exame eletrocardiográfico, como descrito anteriormente, pode detectar a presença de arritmias cardíacas, bem como a presença de alterações no traçado (Filippi, 2011; French, 2016).

Assim, pode-se evidenciar, em decorrência do aumento atrial esquerdo, o prolongamento do tempo da onda $\mathrm{P}$ em alguns casos de DMVM moderada a severa, bem como do complexo QRS, devido à hipertrofia de ventrículo esquerdo. Ademais, pode ser identificado incremento na amplitude da onda R, 
a qual pode estar menor que a onda $S$; depressão do segmento $S T$ e, em casos de derrame pleural ou pericárdico, baixa voltagem (Filippi, 2000; King \& Boag, 2007; Savarino et al., 2012).

Com relação à pressão arterial, é comum que esta esteja alterada em muitos pacientes, dados os mecanismos patofisiológicos que promove maior volemia como forma de melhorar a hemodinâmica (Ettinger et al., 2002).

\section{Tratamentos}

A DMVM pode ser tratada clinicamente como forma de aumentar a qualidade e expectativa de vida por meio da administração de fármacos específicos para o estadiamento do animal, dado que há repercussões hemodinâmicas e comprometimento sistêmico diferentes para cada fase da doença (Häggström et al., 2016; Hemdon et al., 2002; Keene et al., 2019). Nos estágios A e B1, como não há consequências circulatórias, não são recomendados nenhum tipo de tratamento, apenas acompanhamento do animal por meio de ecocardiografias periódicas a cada 6 ou 12 meses (Keene et al., 2019).

Uma possibilidade para retardar a progressão nessas fases é a promoção de atividades físicas regulares aos pacientes como forma de incrementar o tônus parassimpático, tendo em vista que conforme há o avanço no estadiamento, o sistema nervoso simpático tende a ser ativado, de modo promover alterações maléficas (Sabbah, 2012; Valandro et al., 2017). Já no estágio B2, onde já há aumento significativo de átrio esquerdo, recomenda-se a administração de Pimobendan, um inotrópico positivo que atua pouco sobre a fosfodiesterase III, promove maior interação entre o cálcio intracelular e a troponina $\mathrm{C}$, incrementando a capacidade contrátil dos cardiomiócitos e inibe a fosfodiesterase $\mathrm{V}$, resultando na vasodilatação (Keene et al., 2019; Spinosa et al., 2006). Em um estudo conduzido por Keene et al. (2019) utilizando dois grupos de cães, um controle com 180 animais para o qual se administrou placebo, tendo sido um deles excluído, e outro com o mesmo número dessa população composto por indivíduos tratados com a Pimobendan, demostrou que os animais acometidos pela DMVM tratados apenas com administração do fármaco em questão apresentaram prolongamento do período pré-clínico da doença em até 15 meses.

No estágio C, em alguns casos há necessidade de internação, cujos objetivos são: a manutenção do débito cardíaco adequado de modo a promover perfusão adequada a todos os tecidos e a redução da congestão para facilitar o processo de oxigenação (DeFrancesco, 2013; Keene et al., 2019). Assim, devese promover conforto respiratório ao enfermo, colocando-o em decúbito esternal com a cabeça levemente elevada e aumentando a oferta de oxigênio a partir da oxigenoterapia, que poderá ser associada à sedação leve para manejo do animal agitado, utilizando preferencialmente butorfanol (DeFrancesco, 2013; Keene et al., 2019). Não obstante, para controle do edema pulmonar e regulação do débito cardíaco, administra-se diuréticos, preferencialmente furosemida por via intravenosa (IV) ou intramuscular (IM) em bolus ou infusão, sempre avaliando a respiração, bem como Pimobendan e inibidores de ECA ( $\underline{\text { Häggström et al., 2016}}$; Keene et al., 2019). Em casos de edema pulmonar refratário ao tratamento convencional, podemos conduzir a terapia com nitroprussiato de sódio ou outros vasodilatadores, e nos casos de falha circulatória considerável, notada pela hipotensão, podemos administrar Dobutamina (Ettinger et al., 2002; Keene et al., 2019). Para controle da doença em casa, pode-se utilizar, por via oral (VO) Pimobendan; inibidores de ECA, como o benazepril; diurético, preferencialmente a furosemida e a espirolactona, sendo este antagonista de aldosterona; anti-arrítmicos, em casos específicos (Bernay et al., 2010; Keene et al., 2019).

Com ração ao estágio $\mathrm{D}$, ou terminal, tem-se o mesmo protocolo, exceto que alguns autores recomendam a administração de broncodilatadores, inibidores de Fosfodiesterase IV e associação de outros diuréticos em conjunto com outros vasodilatadores caso necessário, tais como a hidralazina e amlodipina (Keene et al., 2019; Reinero et al., 2020). Outra opção para os casos refratários ao uso da Furosemida em doses convencionais é a Torasemida, diurético de alça. Entretanto, uma complicação do uso desta medicação são os efeitos deletérios sobre a função renal do paciente, visto que o uso da Torasemida aumenta o risco de eventos renais em mais de um estudo. Ademais, cabe salientar que a redução do sódio na dieta pode ser benéfica nos estágios avançados, bem como pode ocorrer derrame pleural, pericárdico ou ascite, sendo necessária a drenagem dos conteúdos (Ettinger et al., 2002; Keene et al., 2019; Reinero et al., 2020). 
De acordo com a literatura, a correção cirúrgica da regurgitação mitral é possível, sendo o emprego deste procedimento recomendado quando o paciente recebe estadiamento $\mathrm{C}$, mas também poderá ser executado em animais em estágio B2 e D, a depender do quadro clínico (Orton \& Monnet, 1995). Embora ocorra incremento da expectativa de vida que extrapola os limites da terapia clínica, tal procedimento é pouco usual na Medicina veterinária, dados os altos custos com equipamentos, dificuldades para realização das diferentes técnicas e complicações intraoperatórias, como a lesão do nervo fênico, as provocadas pelo uso do uso de bypass e pós-operatórias, com destaque para a trombose. (Orton et al., 2005; Orton \& Monnet, 1995; Pelosi et al., 2013).

\section{Considerações finais}

Embora notemos avanços no que se diz respeito às pesquisas sobre a degeneração mixomatosa de valva mitral em cães, bem como lançamento de fármacos mais eficientes no mercado, ainda se trata de uma doença que carece de mais estudos para que se compreenda definitivamente todos os processos etiológicos e fisiopatológicos, principalmente na medicina veterinária.

Apesar do novo protocolo de estadiamento, diagnóstico e tratamento representar um grande avanço pela classificação de acordo com as ecocardiografias, as medicações nele descritas e comumente utilizadas são dispendiosas e não promovem cura, tendo por finalidade retardar a progressão da afecção e promover qualidade de vida ao indivíduo.

Embora a correção cirúrgica exista e apresente diferentes técnicas, estas não apresentam efeito satisfatório quando pensamos na possibilidade de usá-las como tratamento de eleição para os estágios aos quais são indicadas, visto que algumas são limitadas a alguns pacientes.

Assim, as pesquisas dos últimos anos relacionadas aos fatores genéticos envolvidos podem ser uma nova proposta de tratamento tanto para animais quanto para humanos, e investimentos nessa área devem ser realizados, já que novas terapias baseadas nos acontecimentos moleculares poderiam ser curativas e oferecidas como opção em detrimento dos atuais. Outra alternativa consiste no investimento para desenvolvimento de novas técnicas que objetivam correção da degeneração da valva mitral.

\section{Referências}

Aikawa, E., Krenning, G., \& Baker, A. H. (2019). Endothelial to mesenchymal transition. Circulation, 104(21), 2525-2532. https://doi.org/10.1016/j.jacc.2018.09.089.

Bernay, F., Bland, J. M., Häggström, J., Baduel, L., Combes, B., Lopez, A., \& Kaltsatos, V. (2010). Efficacy of spironolactone on survival in dogs with naturally occurring mitral regurgitation caused by myxomatous mitral valve disease. Journal of Veterinary Internal Medicine, 24(2), 331-341. https://doi.org/10.1111/j.1939-1676.2009.0467.x.

Boon, J. A. (2011). Veterinary echocardiography. John Wiley \& Sons.

Borgarelli, M., Zini, E., D’Agnolo, G., Tarducci, A., Santilli, R. A., Chiavegato, D., Tursi, M., Prunotto, M., \& Häggström, J. (2004). Comparison of primary mitral valve disease in German Shepherd dogs and in small breeds. Journal of Veterinary Cardiology, 6(2), 27-34. https://doi.org/10.1016/S17602734(06)70055-8.

Boswood, A., Häggström, J., Gordon, S. G., Wess, G., Stepien, R. L., Oyama, M. A., Keene, B. W., Bonagura, J., MacDonald, K. A., \& Patteson, M. (2016). Effect of pimobendan in dogs with preclinical myxomatous mitral valve disease and cardiomegaly: the EPIC study - a randomized clinical trial. Journal of Veterinary Internal Medicine, 30(6), 1765-1779. https://doi.org/10.1016/j.asams.2017.03.008.

Braunwald, E. (2005). Tratado de doenças cardiovasculares. In Tratado de doenças cardiovasculares. Elsevier Brasil.

Buchanan, J. W. (2000). Vertebral scale system to measure heart size in radiographs. Veterinary Clinics of North America Small Animal Practice, 30(2), 379-394. https://doi.org/10.1016/S01955616(00)50027-8.

Chetboul, V., \& Tissier, R. (2012). Echocardiographic assessment of canine degenerative mitral valve disease. Journal of Veterinary Cardiology, 14(1), 127-148. 
https://doi.org/10.1016/j.jvc.2011.11.005.

Cunningham, J. (2011). Tratado de fisiologia veterinária. Guanabara Koogan.

DeFrancesco, T. C. (2013). Management of cardiac emergencies in small animals. Veterinary Clinics: Small Animal Practice, 43(4), 817-842. https://doi.org/10.1016/j.cvsm.2013.03.012.

Eriksson, A. S., Häggström, J., Pedersen, H. D., Hansson, K., Järvinen, A.-K., Haukka, J., \& Kvart, C. (2014). Increased NT-proANP predicts risk of congestive heart failure in Cavalier King Charles spaniels with mitral regurgitation caused by myxomatous valve disease. Journal of Veterinary Cardiology, 16(3), 141-154. https://doi.org/10.1016/j.jvc.2014.05.001.

Ettinger, S. J., Fedlman, E. C., \& Taibo, R. A. (2002). Tratado de medicina interna veterinaria: enfermedades del perro y el gato. Manole.

Filippi, L. H. (2000). O eletrocardiograma na medicina veterinária. Roca Ltda.

French, A. (2016). Pericardial disease. Denis, S., Fuentes, V. L. \& Johnson, L. BSAVA Manual of Canine and Feline Cardiorespiratory Medicine. 2 ed. Inglaterra: British Small Animal Veterinary Association.

Fox, P. R. (2012). Pathology of myxomatous mitral valve disease in the dog. Journal of Veterinary Cardiology, 14(1), 103-126. https://doi.org/10.1016/j.jvc.2012.02.001.

Häggström, J., Andersson, Å. O., Falk, T., Nilsfors, L., OIsson, U., Kresken, J. G., Höglund, K., Rishniw, M., Tidholm, A., \& Ljungvall, I. (2016). Effect of body weight on echocardiographic measurements in 19,866 pure-bred cats with or without heart disease. Journal of Veterinary Internal Medicine, 30(5), 1601-1611. https://doi.org/10.1111/jvim.14569.

Hemdon, W. E., Kittleson, M. D., Sanderson, K., Drobatz, K. J., Clifford, C. A., Gelzer, A., Summerfield, N. J., Linde, A., \& Sleeper, M. M. (2002). Cardiac troponin I in feline hypertrophic cardiomyopathy. Journal of Veterinary Internal Medicine, 16(5), 558-564.

Kealy, J., Graham, J., \& McAllister, H. (2016). Radiografia e ultrassonografia do cão e do gato. Elsevier.

Kealy, J. K., McAllister, H., \& Graham, J. P. (2012). Radiologia e ultrassonografia do cão e do gato (Vol. 1). Elselvier Saunders.

Keene, B. W., Atkins, C. E., Bonagura, J. D., Fox, P. R., Häggström, J., Fuentes, V. L., Oyama, M. A., Rush, J. E., Stepien, R., \& Uechi, M. (2019). ACVIM consensus guidelines for the diagnosis and treatment of myxomatous mitral valve disease in dogs. Journal of Veterinary Internal Medicine, 33(3), 1127-1140. https://doi.org/10.1111/jvim.15488. Acesso em: 2 abr. 2020.

Kim, H.-T., Han, S.-M., Song, W.-J., Kim, B., Choi, M., Yoon, J., \& Youn, H.-Y. (2017). Retrospective study of degenerative mitral valve disease in small-breed dogs: survival and prognostic variables. Journal of Veterinary Science, 18(3), 369. https://doi.org/0.4142/jvs.2017.18.3.369.

King, L. G., \& Boag, A. (2007). BSAVA manual of canine and feline emergency and critical care (Issue Ed. 2). British small animal veterinary association.

Langhorn, R., \& Willesen, J. L. (2016). Cardiac troponins in dogs and cats. Journal of Veterinary Internal Medicine, 30(1), 36-50. https://doi.org/10.1111/jvim.13801.

Lima, G. V., \& Ferreira, F. S. (2017). N-terminal-pro brain natriuretic peptides in dogs and cats: A technical and clinical review. Veterinary World, 10(9), 1072. https://doi.org/10.14202/vetworld.2017.1072-1082.

Lopez-Alvarez, J., Elliott, J., Pfeiffer, D., Chang, Y., Mattin, M., Moonarmart, W., Hezzell, M. J., \& Boswood, A. (2015). Clinical severity score system in dogs with degenerative mitral valve disease. Journal of Veterinary Internal Medicine, 29(2), 575-581. https://doi.org/10.1111/jvim.12544.

Madsen, M. B., Olsen, L. H., Häggström, J., Höglund, K., Ljungvall, I., Falk, T., Wess, G., Stephenson, H., Dukes-McEwan, J., \& Chetboul, V. (2011). Identification of 2 loci associated with development of myxomatous mitral valve disease in Cavalier King Charles Spaniels. Journal of Heredity, 102(Suppl 1), S62-S67. https://doi.org/10.1093/jhered/esr041.

Malcolm, E. L., Visser, L. C., Phillips, K. L., \& Johnson, L. R. (2018). Diagnostic value of vertebral left atrial size as determined from thoracic radiographs for assessment of left atrial size in dogs with 
myxomatous mitral valve disease. Journal of the American Veterinary Medical Association, 253(8), 1038-1045. https://doi.org/10.2460/javma.253.8.1038.

Markby, G. R., Macrae, V. E., Summers, K. M., \& Corcoran, B. M. (2020). Disease severity-associated gene expression in canine myxomatous mitral valve disease is dominated by TGF $\beta$ signaling. Frontiers in Genetics, 11, 372. https://doi.org/10.3389/fgene.2020.00372.

Meurs, K. M., Friedenberg, S. G., Williams, B., Keene, B. W., Atkins, C. E., Adin, D., Aona, B., DeFrancesco, T., Tou, S., \& Mackay, T. (2018). Evaluation of genes associated with human myxomatous mitral valve disease in dogs with familial myxomatous mitral valve degeneration. The Veterinary Journal, 232, 16-19. https://doi.org/10.1016/j.tvj1.2017.12.002.

Orton, E. C., Hackett, T. B., Mama, K., \& Boon, J. A. (2005). Technique and outcome of mitral valve replacement in dogs. Journal of the American Veterinary Medical Association, 226(9), 1508-1511.

Orton, E. C., \& Monnet, E. (1995). Small animal thoracic surgery (Issue V740 ORTs). Wiley Online Library. https://doi.org/10.1002/9781118943427.ch21.

Oyama, M A, \& Levy, R. J. (2010). Insights into serotonin signaling mechanisms associated with canine degenerative mitral valve disease. Journal of Veterinary Internal Medicine, 24(1), 27-36. https://doi.org/10.1111/j.1939-1676.2009.0411.x.

Oyama, Mark A. (2013). Using cardiac biomarkers in veterinary practice. Veterinary Clinics: Small Animal Practice, 43(6), 1261-1272. https://doi.org/10.1016/j.cvsm.2013.07.010.

Oyama, Mark A, Elliott, C., Loughran, K. A., Kossar, A. P., Castillero, E., Levy, R. J., \& Ferrari, G. (2020). Comparative pathology of human and canine myxomatous mitral valve degeneration: 5HT and TGF- $\beta$ mechanisms. Cardiovascular Pathology, 46(1), 107196. https://doi.org/10.1016/j.carpath.2019.107196.

Parker, H. G., \& Kilroy-Glynn, P. (2012). Myxomatous mitral valve disease in dogs: does size matter? Journal of Veterinary Cardiology, 14(1), 19-29. https://doi.org/10.1016/j.jvc.2012.01.006.

Pelosi, A., Anderson, L. K., Paugh, J., Robinson, S., \& Eyster, G. E. (2013). Challenges of cardiopulmonary bypass - a review of the veterinary literature. Veterinary Surgery, 42(2), 119-136. https://doi.org/10.1111/j.1532-950X.2012.01008.x.

Rabkin, E., Aikawa, M., Stone, J. R., Fukumoto, Y., Libby, P., \& Schoen, F. J. (2001). Activated interstitial myofibroblasts express catabolic enzymes and mediate matrix remodeling in myxomatous heart valves. Circulation, 104(21), 2525-2532. https://doi.org/10.1161/hc4601.099489.

Reinero, C., Visser, L. C., Kellihan, H. B., Masseau, I., Rozanski, E., Clercx, C., Williams, K., Abbott, J., Borgarelli, M., \& Scansen, B. A. (2020). ACVIM consensus statement guidelines for the diagnosis, classification, treatment, and monitoring of pulmonary hypertension in dogs. Journal of Veterinary Internal Medicine, 34(2), 549-573. https://doi.org/10.1111/jvim.15725

Richards, J. M., Farrar, E. J., Kornreich, B. G., Moïse, N. S., \& Butcher, J. T. (2012). The mechanobiology of mitral valve function, degeneration, and repair. Journal of Veterinary Cardiology, 14(1), 47-58. https://doi.org/10.1016/j.jvc.2012.01.002.

Sabbah, H. N. (2012). Baroreflex activation for the treatment of heart failure. Current Cardiology Reports, 14(3), 326-333. https://doi.org/10.2217/ica.15.44.

Salguero, X. S., Prandi, D., Llabrés-Díaz, F., Manzanilla, E. G., Badiella, L., \& Bussadori, C. (2019). Heart to spine measurements to detect left atrial enlargement in dogs with mitral insufficiency. Irish Veterinary Journal, 72(1), 1-4. https://doi.org/10.1186/s13620-019-0152-6.

Savarino, P., Borgarelli, M., Tarducci, A., Crosara, S., Bello, N. M., \& Margiocco, M. L. (2012). Diagnostic performance of $\mathrm{P}$ wave duration in the identification of left atrial enlargement in dogs. Journal of Small Animal Practice, 53(5), 267-272. https://doi.org/10.1111/j.17485827.2012.01200.x .

Spinosa, H. S. de S., Górniak, S. L., \& Bernardi, M. M. (2006). Farmacologia aplicada à medicina veterinária. Koogan Guanabara.

Tan, K., Markby, G., Muirhead, R., Blake, R., Bergeron, L., Fici, G., Summers, K., Macrae, V., \& Corcoran, B. (2019). Evaluation of canine 2D cell cultures as models of myxomatous mitral valve degeneration. PloS One, 14(8), e0221126. https://doi.org/10.1371/journal.pone.0221126. 
Terzo, E., Di Marcello, M., McAllister, H., Glazier, B., Lo Coco, D., Locatelli, C., Palermo, V., \& Brambilla, P. G. (2009). Echocardiographic assessment of 537 dogs with mitral valve prolapse and leaflet involvement. Veterinary Radiology \& Ultrasound, 50(4), 416-422. https://doi.org/10.1111/j.1740-8261.2009.01559.x.

Valandro, M. A., Pascon, J. P. E., Pereira, D. T. P., \& Mistieri, M. L. A. (2017). Exercise training of dogs with myxomatous valve disease. Arquivo Brasileiro de Medicina Veterinária e Zootecnia, 69(2), 325-332. https://doi.org/10.1590/1678-4162-9230.

Wolf, J., Gerlach, N., Weber, K., Klima, A., \& Wess, G. (2013). The diagnostic relevance of NT-pro BNP and pro ANP 31-67 measurements in staging of myxomatous mitral valve disease in dogs. Veterinary Clinical Oathology, 42(2), 196-206. https://doi.org/10.1111/vcp.12044.

Histórico do artigo:

Recebido: 1 de janeiro de 2021.

Aprovado: 1 de março de 2021.
Licenciamento: Este artigo é publicado na modalidade Acesso Aberto sob a licença Creative Commons Atribuição 4.0 (CC-BY 4.0), a qual permite uso irrestrito, distribuição, reprodução em qualquer meio, desde que o autor e a fonte sejam devidamente creditados. 